\title{
A SMOOTH, COMPLEX GENERALIZATION OF THE HOBBY-RICE THEOREM
}

\author{
OLEG LAZAREV AND ELLIOTT H. LIEB
}

\begin{abstract}
The Hobby-Rice Theorem states that, given $n$ functions $f_{j}$ on $\mathbb{R}^{N}$, there exists a multiplier $h$ such that the integrals of $f_{j} h$ are all simultaneously zero. This multiplier takes values \pm 1 and is discontinuous. We show how to find a multiplier $h=$ $e^{i g}$ that is infinitely differentiable, takes values on the unit circle, and is such that the integrals of $f_{j} h$ are all zero. We also show the existence of $n$ infinitely differentiable, real functions $g_{j}$ such that the $n$ functions $f_{j} e^{i g_{j}}$ are pairwise orgthogonal.
\end{abstract}

\section{INTRODUCTION}

The purpose of this paper is to generalize the Hobby-Rice Theorem [HR] (see also the later, shorter proof by Pinkus $[\mathrm{P}]$ ), which states that, given $n$ integrable real-valued functions $f_{1}, \ldots, f_{n}$ on $[0,1]$, it is possible to find $0=\alpha_{0}<\alpha_{1}<\cdots<\alpha_{r}<\alpha_{r+1}=1$ (with $r \leq n$ ) such that

$$
\sum_{m=1}^{r+1}(-1)^{m} \int_{\alpha_{m-1}}^{\alpha_{m}} f_{j}(x) d x=0
$$

for all $1 \leq j \leq n$.

That is, the Hobby-Rice Theorem says that there exists $g:[0,1] \mapsto\{0, \pi\}$ such that

$$
\int_{0}^{1} f_{j}(x) e^{i g(x)} d x=0
$$

for $1 \leq j \leq n$. This $g$ is discontinuous, however. The purpose of this paper is to generalize the Hobby-Rice Theorem by letting $g \in C_{c}^{\infty}((0,1) ; \mathbb{R})$. In particular, this implies that if $f_{1}, \ldots, f_{n} \in H^{1}([0,1])$, then $f_{j} e^{i g} \in H^{1}([0,1])$ as well.

If the functions $f_{j}$ are complex-valued, we can consider them to be $2 n$ real-valued functions; thus, (1.1), (1.2) continue to hold except that $r \leq 2 n$. The words 'complex generalization' in our title do not refer to this trivial generalization, but rather, to the fact that the multiplier is a smooth function on the unit circle in the complex plane.

There seems to be no way to adapt the proof of the Hobby-Rice Theorem (which involves a fixed-point argument) to find a smooth $g$. Instead, we start with the discontinuous $g$ given by the Hobby-Rice Theorem, and modify $g$ to get a smooth function.

Date: March 27, 2014.

(C) 2012 by the authors. This paper may be reproduced, in its entirety, for non-commercial purposes.

Work partially supported by NSF grant PHY-0965859 (E.H.L.).

Published in Indiana Univ. Math. Jour. 62, 1133-1141 (2013). DOI: 10.1512/iumj.2013.62.5062. 
Our proof is more complicated than desired, and we offer it as a challenge for simplification. In particular, we have no estimate on the derivatives of $g$, and therefore, we cannot estimate the $H^{1}$ norm of $f_{j} e^{i g}$ in terms of the $H^{1}$ norm of $f_{j}$. Vermont Rutherfoord has extended our results in $[\mathrm{R}$.

Our main theorem is the following.

Theorem 1.1. Let $f_{1}, \ldots, f_{n} \in L^{1}([0,1])$ be real-valued. Then, there exists $g \in$ $C_{c}^{\infty}(0,1)$, real-valued, such that

$$
\int_{0}^{1} f_{j}(x) e^{i g(x)} d x=0
$$

for $1 \leq j \leq n$.

As noted before, the validity of Theorem 1.1 for real-valued functions implies its validity for complex-valued functions. The following is also an important corollary of Theorem 1.1.

Corollary 1.2. If the functions in Theorem 1.1 are taken to be in $L^{1}\left(\mathbb{R}^{N}\right)$ instead of $L^{1}([0,1])$, then there exists a real-valued $g \in C_{c}^{\infty}\left(\mathbb{R}^{1}\right)$ so that

$$
\int_{\mathbb{R}^{N}} f_{j}(x) e^{i g\left(x_{1}\right)} d x=0
$$

for $1 \leq j \leq n$.

Proof of the corollary. First, to reduce the $L^{1}\left(\mathbb{R}^{1}\right)$ version to the $L^{1}([0,1])$ version, we map $x$ to $\left(e^{-x}+1\right)^{-1}$ so that $(-\infty, \infty)$ maps to $[0,1]$, and we absorb the Jacobian into the functions on $[0,1]$. That is, we have

$$
\int_{\mathbb{R}^{1}} f_{j}(x) \exp \left(i g\left(\left(e^{-x}+1\right)^{-1}\right)\right) d x=\int_{0}^{1} \frac{f_{j}\left(-\ln \left(x^{-1}-1\right)\right)}{x(1-x)} e^{i g(x)} d x .
$$

We then apply Theorem 1.1 to $f_{j}\left(-\ln \left(x^{-1}-1\right)\right) /(x(1-x))$ to find $g(x)$. The solution to the problem on $\mathbb{R}^{1}$ will then be $g\left(\left(e^{-x}+1\right)^{-1}\right)$.

To reduce the $L^{1}\left(\mathbb{R}^{N}\right)$ version to the $L^{1}\left(\mathbb{R}^{1}\right)$ version, we let

$$
F_{j}(x)=\int_{\mathbb{R}^{N-1}} f_{j}\left(x, x_{2}, \ldots, x_{N}\right) d x_{2} \cdots d x_{N}
$$

and then apply the $L^{1}\left(\mathbb{R}^{1}\right)$ version of Theorem 1.1 to $F_{j}(x)$, obtaining $g(x) . g\left(x_{1}, \ldots, x_{N}\right)=$ $g\left(x_{1}\right)$. This function does not have compact support in $\mathbb{R}^{N}$, but is in $C^{\infty}\left(\mathbb{R}^{N}\right)$ and is bounded.

Theorem 1.1 and Corollary 1.2 can be used as follows to smoothly orthogonalize any set of any $n$ functions without changing their moduli.

Corollary 1.3. Let $f_{1}, \ldots, f_{n} \in L^{2}\left(\mathbb{R}^{N}\right)$. Then, there exist $n$ real functions $g_{1}, \ldots, g_{n} \in$ $C_{c}^{\infty}\left(\mathbb{R}^{1}\right)$ such that the $n$ functions $\varphi_{j}(x):=f_{j}(x) \exp \left\{i g_{j}\left(x_{1}\right)\right\}$ are pairwise orthogonal. 
Proof of Corollary. Use Theorem 1.1 as follows.

Choose $g_{n}(x)=0$. Choose $g_{n-1}$ so that $\int f_{n-1}^{*} \varphi_{n} e^{-i g_{n-1}}=0$. Next, choose $g_{n-2}$ so that the two integrals $\int f_{n-2}^{*} \varphi_{j} e^{-i g_{n-2}}=0$ for $j=n$ and $j=n-1$. Similarly, determine $g_{n-3}$ so that three integrals vanish, and so on, finishing with $\int f_{1}^{*} \varphi_{j} e^{-i g_{1}}=0$ for $j=2, \ldots n$.

1.1. Acknowledgements. The authors are grateful to Rupert Frank for helpful comments and to Robert Schrader for motivating our work and for his constant encouragement. The motivation for our work is to prove a necessary ingredient in a proof of a theorem in the 'density functional theory' of quantum mechanics [LS]. Specifically, we want to know that, given a one-particle density and a one-particle current density, we can find $n$ orthonormal functions in $H^{1}\left(\mathbb{R}^{3}\right)$ such that the sums of their individual densities and currents equal the given values.

\section{Proof of Theorem 1.1}

To prove Theorem 1.1, we start with the following special case of that theorem, which will be used in an inductive proof.

Theorem 2.1. Suppose the conditions of Theorem 1.1 hold, but with the additional assumption that there exists $p \in(0,1)$ such that the functions $f_{1}, \ldots, f_{n}$ are linearly independent on $[0, p]$ and on $[p, 1]$. Then, the conclusion of Theorem 1.1 is true.

We begin by proving Theorem 2.1 ,

Proof. By the Hobby-Rice theorem for $f_{1}, \ldots, f_{n}$ restricted to $[0, p]$, there exist $\left\{\alpha_{m}\right\}_{m=1}^{r}$, $r \leq n, 0=\alpha_{0}<\alpha_{1}<\cdots<\alpha_{r}<\alpha_{r+1}=p$ such that, for $1 \leq j \leq n$,

$$
\sum_{m=1}^{r+1}(-1)^{m} \int_{\alpha_{m-1}}^{\alpha_{m}} f_{j}(x) d x=0 .
$$

Similarly, by the Hobby-Rice theorem for $f_{1}, \ldots, f_{n}$ restricted to $[p, 1]$, there exist $\left\{\beta_{m}\right\}_{m=1}^{s}, s \leq n, p=\beta_{0}<\beta_{1}<\cdots<\beta_{s}<\beta_{s+1}=1$ such that, for $1 \leq j \leq n$,

$$
\sum_{m=1}^{s+1}(-1)^{m} \int_{\beta_{m-1}}^{\beta_{m}} f_{j}(x) d x=0 .
$$

Therefore, we have

$$
0=\left(\sum_{m=1}^{r+1}(-1)^{m} \int_{\alpha_{m-1}}^{\alpha_{m}} f_{j}(x) d x\right)+i\left(\sum_{m=1}^{s+1}(-1)^{m} \int_{\beta_{m-1}}^{\beta_{m}} f_{j}(x) d x\right)
$$

for $1 \leq j \leq n$. Writing the $\pm 1, \pm i$ in complex polar coordinates, we have

$$
\int_{0}^{1} f_{j}(x) e^{i g_{0}(x)} d x=0
$$


where

$$
g_{0}(x)= \begin{cases}0 & : x \in\left[\alpha_{m}, \alpha_{m+1}\right), m \text { odd } \\ \pi & : x \in\left[\alpha_{m}, \alpha_{m+1}\right), m \text { even } \\ \pi / 2 & : x \in\left[\beta_{m}, \beta_{m+1}\right), m \text { odd } \\ 3 \pi / 2 & : x \in\left[\beta_{m}, \beta_{m+1}\right), m \text { even }\end{cases}
$$

Note that $e^{i g_{0}}$ is real to the left of $p$ and imaginary to the right of $p$.

Even though the integrals in (2.4) are zero, the problem is that $g_{0}$ is not smooth (or even continuous) at the points $\alpha_{m}$ or $\beta_{m}$. Therefore, for $\varepsilon>0$, we now consider an approximation $g_{\varepsilon} \in C_{c}^{\infty}((0,1) ; \mathbb{R})$ to $g_{0}$ such that $g_{\varepsilon}$ differs from $g_{0}$ only in $\varepsilon$-intervals around the points $\alpha_{m}$ and $\beta_{m}$, and moreover, such that $0 \leq g_{\varepsilon}(x)<2 \pi$. Since the functions $f_{j}$ are integrable, for $1 \leq j \leq n$

$$
\left|\int_{0}^{1} f_{j}(x) e^{i g_{\varepsilon}(x)} d x\right|=\left|\int_{0}^{1} f_{j}(x)\left(e^{i g_{\varepsilon}(x)}-e^{i g_{0}(x)}\right) d x\right| \rightarrow 0 \quad \text { as } \varepsilon \rightarrow 0 .
$$

We would like to replace $g_{0}$ by $g_{\varepsilon}$, which will be smooth, but then the integrals in (2.4) will no longer be equal to zero. Therefore, we shall further perturb $g_{\varepsilon}$ by smooth functions so as to make the integrals in (2.4) zero again.

To do so, consider functions $h_{1}, \ldots, h_{n} \in C_{c}^{\infty}((0, p) ; \mathbb{R})$ and $h_{n+1}, \ldots, h_{2 n} \in$ $C_{c}^{\infty}((p, 1) ; \mathbb{R})$. For such functions $h$, define $F_{\varepsilon}^{j}: \mathbb{R}^{2 n} \mapsto \mathbb{C}$ by

$$
F_{\varepsilon}^{j}(u)=\int_{0}^{1} f_{j}(x) \exp \left(i g_{\varepsilon}(x)+i \sum_{m=1}^{2 n} u_{m} h_{m}(x)\right) d x
$$

where $u=\left(u_{1}, \ldots, u_{2 n}\right) \in \mathbb{R}^{2 n}$. Also, define $Q_{\varepsilon}: \mathbb{R}^{2 n} \mapsto \mathbb{R}^{2 n}$ by

$$
Q_{\varepsilon}(u)=\left(\Im\left(F_{\varepsilon}^{1}(u)\right), \ldots, \Im\left(F_{\varepsilon}^{n}(u)\right), \Re\left(F_{\varepsilon}^{1}(u)\right), \ldots, \Re\left(F_{\varepsilon}^{n}(u)\right)\right) .
$$

Note that $Q_{0}(0)=0$, because of (2.4). If $\|\cdot\|$ is the Euclidean norm on $\mathbb{R}^{2 n}$, then, by $(2.6),\left\|Q_{\varepsilon}(0)\right\| \rightarrow 0$ as $\varepsilon \rightarrow 0$.

To show that we can find $u$ to compensate the $g_{0}$ to $g_{\varepsilon}$ perturbation, we start by showing that $u \mapsto Q_{0}(u)$ is invertible in a neighborhood of $u=0$ for some $h_{1}, \ldots, h_{2 n}$. To do this, we use the inverse function theorem, which requires showing that the Jacobian matrix $D Q_{0}(0)$ has a non-zero determinant. Note that the partial derivatives are

$$
\frac{\partial F_{0}^{j}}{\partial u_{k}}=i \int_{0}^{1} f_{j}(x) h_{k}(x) \exp \left(i g_{0}(x)+i \sum_{m=1}^{2 n} u_{m} h_{m}(x)\right) d x,
$$

which are continuous in $u$ on $\mathbb{R}^{2 n}$. In particular, at $u=0$, the partial derivatives are

$$
\left.\frac{\partial F_{0}^{j}}{\partial u_{k}}\right|_{u=0}=i \int_{0}^{1} f_{j}(x) h_{k}(x) e^{i g_{0}(x)} d x
$$

Also, note that we constructed $g_{0}$ so that $i e^{g_{0}}$ is imaginary in $[0, p]$, the support of $h_{1}, \ldots, h_{n}$, and, similarly, so that $i e^{g_{0}}$ is real in $[p, 1]$, the support of $h_{n+1}, \ldots, h_{2 n}$. 
Therefore, $\left.\left(\partial F_{0}^{j} / \partial u_{k}\right)\right|_{u=0}$ is imaginary for $1 \leq k \leq n$ and real for $n+1 \leq k \leq 2 n$. As a result, the Jacobian matrix is block-diagonal:

$$
D Q_{0}(0)=\left(\begin{array}{cc}
A & 0 \\
0 & B
\end{array}\right)
$$

where $A, B$ are $n \times n$ matrices with entries

$$
\begin{aligned}
& A_{j k}=\left.\Im\left(\frac{\partial F_{0}^{j}}{\partial u_{k}}\right)\right|_{u=0}=\int_{0}^{p} f_{j}(x) h_{k}(x) e^{i g_{0}(x)} d x \\
& B_{j k}=\left.\Re\left(\frac{\partial F_{0}^{j}}{\partial u_{k}}\right)\right|_{u=0}=i \int_{p}^{1} f_{j}(x) h_{k+n}(x) e^{i g_{0}(x)} d x .
\end{aligned}
$$

By Proposition 2.2, which we will prove later, there do exist real-valued functions $h_{1}$, $\ldots, h_{n} \in C_{c}^{\infty}((0, p) ; \mathbb{R})$ and $h_{n+1}, \ldots, h_{2 n} \in C_{c}^{\infty}((p, 1) ; \mathbb{R})$ such that $\operatorname{det} D Q_{0}(0) \neq 0$. Proposition 2.2 is where we use the condition of Theorem 2.1 that the functions $f$ are linearly independent in $[0, p]$ and in $[p, 1]$. Also, since the determinant of a matrix is a continuous function of the matrix entries, we may assume that there exists $\varepsilon_{0}>0$ such that the functions $h$ vanish in $\varepsilon_{0}$-intervals of the points $\alpha_{m}$ and $\beta_{m}$, and that $\operatorname{det} D Q_{0}(0)$ is still not equal to zero. While this requirement may seem like a large perturbation of the functions $h$, it is a small perturbation of integrals defining $A_{j k}$ and $B_{j k}$.

The crucial point about our construction is that for $\varepsilon<\varepsilon_{0}$, the supports of $g_{\varepsilon}-g_{0}$ and the functions $h$ are now disjoint! By using this fact, we will show that $Q_{\varepsilon}$ can be written as

$$
Q_{\varepsilon}(u)=Q_{0}(u)+C(\varepsilon), \quad \text { for } \varepsilon<\varepsilon_{0},
$$

where $C(\varepsilon)$ is independent of $u$ and $\|C(\varepsilon)\| \rightarrow 0$ as $\varepsilon \rightarrow 0$. Thus, we have decoupled the smoothing of the jump in $g_{0}$ (which is the $C(\varepsilon)$ term) from the perturbation of constant parts of $g_{0}$ (which is the $Q_{0}(u)$ term). It is then easy to use the latter to compensate for the former.

To prove (2.13), we rewrite $F_{j}^{\varepsilon}$ as

$$
F_{j}^{\varepsilon}(u)=F_{j}^{0}(u)+\left(F_{j}^{\varepsilon}(u)-F_{j}^{0}(u)\right) .
$$

Note that $F_{j}^{\varepsilon}(u)-F_{j}^{0}(u)$ does not depend on $u$ when $\varepsilon<\varepsilon_{0}$. This is because

$$
F_{j}^{\varepsilon}(u)-F_{j}^{0}(u)=\int_{0}^{1} f_{j}(x) \exp \left(i \sum_{m=1}^{2 n} u_{m} h_{m}(x)\right)\left(e^{i g_{\varepsilon}(x)}-e^{i g_{0}(x)}\right) d x
$$

and $e^{i g_{\varepsilon}(x)}-e^{i g_{0}(x)}$ is non-zero only on $\varepsilon$-intervals centered at the points $\alpha_{m}$ and $\beta_{m}$. On the other hand, the functions $h$ vanish on $\varepsilon_{0}$-intervals centered at the points $\alpha_{m}$ and $\beta_{m}$. Therefore, if $C(\varepsilon) \in \mathbb{R}^{2 n}$ denotes the real and imaginary parts of $F_{j}^{\varepsilon}(u)-F_{j}^{0}(u)$, we have (2.13). Also, note that we have $\|C(\varepsilon)\| \rightarrow 0$ as $\varepsilon \rightarrow 0$ because of (2.6) $)$.

We next explain how to use the inverse function theorem to carry out the compensation. Since $Q_{0}(0)=0$ and $\operatorname{det} D Q_{0}(0) \neq 0$ and the partial derivatives of $Q_{0}$ 
are continuous, the theorem guarantees the existence of a ball $B_{R}(0) \subset \mathbb{R}^{2 n}$ of radius $R>0$ around 0 such that, when $y \in B_{R}(0)$, there exists $u \in \mathbb{R}^{2 n}$ such that $Q_{0}(u)=y$.

As stated in (2.13), for $\varepsilon<\varepsilon_{0}$, we see that $Q_{\varepsilon}$ and $Q_{0}$ differ by a constant depending only on $\varepsilon$. Thus, for every $y \in B_{R}\left(Q_{\varepsilon}(0)\right)$, there exists $u \in \mathbb{R}^{2 n}$ such that $Q_{\varepsilon}(u)=y$. Now take $0<\varepsilon<\varepsilon_{0}$ small enough so that $\|C(\varepsilon)\|<R$. Then $Q_{\varepsilon}(0)=C(\varepsilon)$ and $\left\|Q_{\varepsilon}(0)-0\right\|=\|C(\varepsilon)\|<R$. Therefore, $0 \in B_{R}\left(Q_{\varepsilon}(0)\right)$, and so there exists $u \in \mathbb{R}^{2 n}$ such that $Q_{\varepsilon}(u)=0$. For such $u$, let $g(x)=g_{\varepsilon}(x)+\sum_{m=1}^{2 n} u_{m} h_{m}(x)$. Then,

$$
\int_{0}^{1} f_{j}(x) e^{i g(x)} d x=0
$$

for $1 \leq j \leq n$, as desired. Note that $g \in C_{c}^{\infty}((0,1) ; \mathbb{R})$ since $g_{\varepsilon} \in C_{c}^{\infty}((0,1) ; \mathbb{R})$ for $\varepsilon>0$, and we chose $h_{k} \in C_{c}^{\infty}((0, p) ; \mathbb{R})$ and $h_{n+k} \in C_{c}^{\infty}((p, 1) ; \mathbb{R})$.

To complete the proof of Theorem 2.1, it is necessary to prove Proposition 2.2, which was used in the proof above.

Proposition 2.2. If the functions satisfy the conditions of Theorem 2.1, there exist real-valued $h_{1}, \ldots, h_{n} \in C_{c}^{\infty}((0, p) ; \mathbb{R}), h_{n+1}, \ldots, h_{2 n} \in C_{c}^{\infty}((p, 1) ; \mathbb{R})$ such that $\operatorname{det} D Q_{0}(0) \neq 0$.

Proof. Our proof is by contradiction. Suppose that $\operatorname{det} D Q_{0}(0)=0$ for every $h_{1}, \ldots, h_{n} \in$ $C_{c}^{\infty}((0, p) ; \mathbb{R}), h_{n+1}, \ldots, h_{2 n} \in C_{c}^{\infty}((p, 1) ; \mathbb{R})$. Then, either $\operatorname{det} A=0$ for every $h_{1}, \ldots$, $h_{n}$ or $\operatorname{det} B=0$ for every $h_{n+1}, \ldots, h_{2 n}$; otherwise, if $\operatorname{det} A \neq 0$ for some $h_{1}, \ldots, h_{n}$ and $\operatorname{det} B \neq 0$ for some $h_{n+1}, \ldots, h_{2 n}$, then $\operatorname{det} D Q_{0}(0)=\operatorname{det} A \operatorname{det} B \neq 0$ for such $h_{1}, \ldots, h_{2 n}$, which is a contradiction. Having assumed linear independence of the functions $f$ both to the left of $p$ and to the right of $p$, let us assume, without loss of generality, that $\operatorname{det} A=0$ for every $h_{1}, \ldots, h_{n}$. We will show that there exist $a_{1}, \ldots, a_{n} \in \mathbb{R}$ (not all 0 ) such that $\sum_{j=1}^{n} a_{j} f_{j}(x)=0$ almost everywhere on $[0, p]$.

Suppose we let $h_{1}$ vary and fix the rest of the functions $h$. Then, for all $h_{1}$, we have

$$
\sum_{j=1}^{n} C_{j, 1} \int_{0}^{p} f_{j}(x) h_{1}(x) e^{i g_{0}(x)} d x=\operatorname{det} A=0,
$$

where $C_{j, 1}$ is the $(j, 1)$-cofactor of $A$. We can assume that at least one of the cofactors is non-zero, since otherwise we can look at a smaller matrix. Note that these cofactors depend only on $h_{2}, \ldots, h_{n}$, and not on $h_{1}$. Since (2.17) holds for all $h_{1} \in C_{c}^{\infty}((0, p) ; \mathbb{R})$,

$$
\sum_{j=1}^{n} C_{j, 1} f_{j}(x)=0
$$

almost everywhere on $[0, p]$, which contradicts independence.

We now use Theorem 2.1 to complete the proof of Theorem 1.1. First, we use the following proposition to split the theorem into two cases.

Proposition 2.3. Given n Lebesgue-measurable functions on $[0,1]$, exactly one of the two following cases holds: 
(1) There exists $p \in(0,1)$ such that $f_{1}, \ldots, f_{n}$ are linearly independent almost everywhere on $[0, p]$ and on $[p, 1]$.

(2) There exists $q \in[0,1]$ such that $f_{1}, \ldots, f_{n}$ are linearly dependent almost everywhere on $[0, q]$ and on $[q, 1]$.

Proof. To see that one of these cases holds, consider

$$
\begin{aligned}
& L=\sup \left\{x_{0} \in[0,1]: \sum_{j=1}^{n} a_{j} f_{j}(x)=0 \text { a.e. on }\left[0, x_{0}\right] \text { for some }\left(a_{1}, \ldots, a_{n}\right) \neq 0\right\} \\
& R=\inf \left\{x_{0} \in[0,1]: \sum_{j=1}^{n} b_{j} f_{j}(x)=0 \text { a.e. on }\left[x_{0}, 1\right] \text { for some }\left(b_{1}, \ldots, b_{n}\right) \neq 0\right\} .
\end{aligned}
$$

Note that the extremizers $L$ and $R$ are actually reached; in other words, there exists $\left(a_{1}, \ldots, a_{n}\right)$ such that $\sum_{j=1}^{n} a_{j} f_{j}(x)=0$ on $[0, L]$, and similarly for $[R, 1]$. This is because, for every $x_{0}<L$, the set of relations $\left(a_{1}, \ldots, a_{n}\right)$ such that $\sum_{j=1}^{n} a_{j} f_{j}(x)=0$ on $\left[0, x_{0}\right]$ is a vector space whose dimension is positive and finite. As $x$ increases, this dimension can only decrease, since if $\sum_{j=1}^{n} a_{j} f_{j}(x)=0$ on $\left[0, x_{0}\right]$, then this is also true on $[0, y]$ for any $y<x_{0}$. Now consider the relations that form a basis for this vector space. Since the dimension of the vector space only decreases, one of these relations must hold until $L$, which means that a relationship holds almost everywhere on $[0, L]$ as desired.

To prove the claim that either Case 1 or Case 2 must hold, note that if $L<R$, then Case 1 holds for any $p$ satisfying $L<p<R$; and if $L \geq R$, then Case 2 holds for any $q$ satisfying $R \leq q \leq L$.

We prove Theorem 1.1 by induction on $n$, the number of functions. If $n=1$, then the linear independence condition of Theorem 2.1 holds for some $p$ because $f_{1}$ is not identically zero.

Suppose that Theorem 1.1 holds for $n-1$ functions on $[0,1]$. Consider $n$ functions $f_{1}, \ldots, f_{n}$. By Proposition 2.3 , this set of functions belongs either to Case 1 or Case 2. Note that Case 1 is exactly appropriate for Theorem 2.1, and so Theorem 1.1 holds in this case.

Now suppose that the $n$ functions belong to Case 2. Therefore, there are no more than $n-1$ linearly independent functions on both sides of $q$ (even if $q=0$ or 1 ). By the induction hypothesis, we can apply Theorem 1.1 to $[0, q]$ and to $[q, 1]$ (via change-of-variables). Then, there exist $g_{1} \in C_{c}^{\infty}((0, q) ; \mathbb{R})$ and $g_{2} \in C_{c}^{\infty}((q, 1) ; \mathbb{R})$ such that

$$
\int_{0}^{q} f_{j}(x) e^{i g_{1}(x)} d x=0 \quad \text { for } 1 \leq j \leq n
$$

and

$$
\int_{q}^{1} f_{j}(x) e^{i g_{2}(x)} d x=0 \quad \text { for } 1 \leq j \leq n
$$


Let $g:[0,1] \mapsto[0,1]$ be such that $g \uparrow_{[0, q]}=g_{1}$ and $g \uparrow_{[q, 1]}=g_{2}$. Then, we have

$$
\int_{0}^{1} f_{j}(x) e^{i g(x)} d x=0 \quad \text { for } 1 \leq j \leq n
$$

as desired. Note that $g \in C_{c}^{\infty}((0,1) ; \mathbb{R})$ because $g_{1}(q)=g_{2}(q)=0$ and all derivatives of $g_{1}, g_{2}$ are zero at $q$ (by the compact support condition for $g_{1}$ and $g_{2}$ ). Therefore, Theorem 1.1 holds by induction.

\section{REFERENCES}

[HR] Charles R. Hobby and John R. Rice, A Moment Problem in L1 Approximation, Proc. Amer. Math. Soc., 16 , No. 4 (1965). http://dx.doi.org/10.2307/2033900 MR 0178292 (31 \#2550),

[LS] Elliott H. Lieb and Robert Schrader, Current-Densities in Density Functional Theory, Phys.Rev. A bf 88, 032516 (2012). http://dx.doi.org/10.1103/PhysRevA.88.032516.

[P] Allan Pinkus, A Simple Proof of the Hobby-Rice Theorem, Proc. Amer. Math. Soc., 20, No. 1, 82-84 (1976). http://dx.doi.org/10.1090/S0002-9939-1976-0425470-0, MR 0425470 (54 \#13425).

[R] Vermont Rutherfoord, On the Lazarev-Lieb Extension of the Hobby-Rice Theorem, http://arxiv.org/abs/1212.5759.

Oleg Lazarev, Department of Mathematics, Princeton University, Washington Road, Princeton, NJ, 08542, USA.

Present address: Department of Mathematics, Stanford University, Building 380, StANFORd, CA 94305, USA

E-mail address: olazarev@stanford.edu

Elliott H. Lieb, Departments of Mathematics and Physics, Princeton University, Washington Road, Princeton, NJ 08544, USA

E-mail address: lieb@princeton.edu 